\title{
ÍNDICE DE CONSUMO E EFICIÊNCIA DO USO DA ÁGUA EM EUCALIPTO, SUBMETIDO A DIFERENTES TEORES DE ÁGUA EM CONVIVÊNCIA COM BRAQUIÁRIA
}

\author{
Wilson da Silva ${ }^{1}$ \\ Tocio Sediyama ${ }^{2}$ \\ Antônio Alberto da Silva ${ }^{2}$ \\ Antônio Américo Cardoso ${ }^{3}$
}

\begin{abstract}
RESUMO
Foram avaliados, em casa de vegetação, os índices de consumo e a eficiência do uso da água em mudas de Eucalyptus citriodora e E. grandis, cultivadas em solo com três teores de água, associadas ou não com Brachiaria brizantha. Utilizou-se o delineamento em blocos ao acaso, com quatro repetições, em arranjo fatorial $(2 \times 3 \times 4+3 \times 3)$ : duas espécies (E. citriodora e $E$. grandis), três teores de água $(20,23$ e $26 \%)$ e quatro diferentes quantidades de plantas de $B$. brizantha $(0,1,2$ e 3 plantas/vaso), mais três diferentes quantidades de $B$. brizantha (1, 2 e 3 plantas), associadas aos três teores de água. Maior crescimento do E. citriodora, E. grandis e $B$. brizantha foi observado em solo com o maior teor de umidade (26\%). A convivência de B. brizantha com $E$. citriodora ou E. grandis, em um mesmo vaso, reduziu o acúmulo de biomassa seca do eucalipto, independente do teor de água no solo. Maiores teores de água no solo proporcionaram maior ICA, que está diretamente relacionado a maior acúmulo de biomassa seca pelos eucaliptos e $B$. brizantha. A eficiência do uso de água do eucalipto foi alta (282 g de água/g de biomassa seca produzida), semelhante ao observado por $B$. brizantha (265), ambas espécies estão dentro da faixa de plantas eficientes ao uso da água, independente do teor de água no solo.

Palavras-chave: mudas, E. grandis, E. citriodora, B. brizantha, coeficiente transpiratório
\end{abstract}

CONSUMPTION AND WATER EFFICIENCY USE INDEX BY Eucalyptus citriodora AND E. grandis PLANTS CULTIVATED IN POTS CONTAINING SOIL WITH THREE WATER CONTENTS IN THE SOIL JOINTLY WITH DIFFERENT Brachiaria brizantha POPULATIONS

\begin{abstract}
The objective of this study was to evaluate water efficient use of, E. citriodora and E. grandis amount of cultivated in subtract with three water contents, jointly with four amount of Brachiaria brizantha plant. Treatments were arranged in factorial design, with four replications, in a randomised blocks $(2 \times 3 \times 4+3+3)$ : two species of eucalyptus (E. citriodora and E. grandis), three water contents (20, 23 and 26\%) and four B. brizantha populations (0,1, 2 and 3 plants/pot) and three plus $B$. brizantha (1, 2 and 3 plants/pot) associated to three contents of water contents in the soil. The highest growing of E. citriodora, E. grandis and B. brizantha was observed in a soil with the highest moisture content $(26 \%)$. B. brizantha association with E. citriodora or $E$. grandis, in the same pot, reduced the eucalyptus dry biomass accumulation regardless of water content in the soil. Higher water contents in the soil allowed higher Water Consumption Index, which is directly related to the higher accumulation of dry biomass by eucalyptus and $B$. brizantha Eucalyptus water use efficiency (transpiratory coefficient) was high (282 $\mathrm{g}$ water/g of produced dry matter), similar to observed for B. brizantha (265). They are in the range of efficient plants in water use, regardless of water content in the soil.
\end{abstract}

Keywords: plants cutting, E. grandis, E. citriodora, B. brizantha, transpiratory coefficient

\footnotetext{
${ }^{1}$ Doutor em Fitotecnia, Dep. de Fitotecnia da UFV, Campus Universitário, CEP 36571.000 Viçosa, MG; silvawilson@hotmsil.com

${ }^{2}$ Prof. Dr. do Dep. Fitotecnia da UFV, E-mail: aasilva@ufv.br

${ }^{3}$ Prof. Dr. Aposentado do Dep. Fitotecnia da UFV e bolsista do CNPq, E-mail: aacardoso@ufv.br

Recebido para publicação: 27/02/2003

Aceito para publicação: 03/09/2004
} 


\section{INTRODUÇÃO}

As áreas reflorestadas com eucalipto, na maioria das vezes, estão estabelecidas principalmente em áreas de cerrado, onde os solos apresentam baixa fertilidade, estando sujeitos a longos períodos de déficit hídrico. Em geral, a temperatura, a insolação e a radiação solar não constituem impedimentos ao crescimento das plantas durante grande parte do ano (Goedert et al., 1980).

Algumas espécies de Eucalyptus podem suportar solos com elevada acidez (Vale, 1982), com baixa fertilidade (Barros \& Novais, 1990) e regime hídrico irregular (Gomes, 1994). Nessas condições a produtividade pode ser aumentada, consideravelmente, utilizando-se técnicas silviculturais, tais como: adubação (Novais et al. 1990); escolha do espaçamento adequado (Gomes, 1994); e controle de plantas daninhas (Silva, 1993).

A adaptação de espécies de eucalipto em ambientes em que ocorre limitação da quantidade e, ou, da distribuição de chuvas pode comprometer a sobrevivência e o crescimento das plantas e, por isso, é preciso selecionar as espécies que apresentam mecanismos eficientes de uso da água e de nutrientes (Jones \& Wilkins, 1971).

Algumas espécies do gênero Eucalyptus, a exemplo de outras espécies arbóreas, apresentam mecanismos de economia de água (Pryor, 1976). Alguns desses mecanismos envolvem a presença de tecido foliar coriáceo espessado, alinhamento vertical das folhas, fechamento rápido dos estômatos, baixas taxas de transpiração e elevada razão raiz/parte aérea.

Há grande evidência de que a eficiência do uso de água pelas plantas varia entre as espécies no mesmo ambiente, entre diferentes condições climáticas numa mesma cultura, entre sítios e estação do ano (Taylor \& Willatt, 1983). A adaptação de espécies aos efeitos do clima pode ajudar no manejo de água em condições de diferente umidade; razão pela qual o uso da água reflete a complexidade de fatores envolvidos na interação planta/ambiente. De acordo com Olbrich et al. (1993), o conhecimento da eficiência do uso da água ajudaria selecionar melhores as espécies de eucalipto para determinadas condições.

Este trabalho teve por objetivo avaliar, em casa de vegetação, o índice de consumo e eficiência no uso da água por mudas de Eucalyptus citriodora Hook e E. grandis Benth. W. Hill (ex Maiden), cultivadas em solo com três teores de água e associação com Brachiaria brizantha Stapf.

\section{MATERIAL E MÉTODOS}

$\mathrm{O}$ experimento foi conduzido em condições de casa de vegetação, no Departamento de Fitotecnia da Universidade Federal de Viçosa, Viçosa, MG. O solo utilizado no experimento foi um Argissolo Vermelho-Amarelo câmbico apresentando textura argilo-arenosa, com $38 \%$ de areia grossa, $15 \%$ de areia fina, $10 \%$ de silte e $37 \%$ de argila (EMBRAPA, 1997); carbono orgânico 3,0\% (Defelipo \& Ribeiro, 1981) e $\mathrm{pH} 5,8$, tendo sido coletado sob floresta nativa, na profundidade de $0-20 \mathrm{~cm}$ em Viçosa, MG. A curva característica de umidade desse solo (Figura 1), foi determinada no laboratório utilizando-se o aparelho de membrana de pressão de Richards (1949). Essa curva foi utilizada para a determinação da quantidade de água a ser adicionada em cada vaso. 


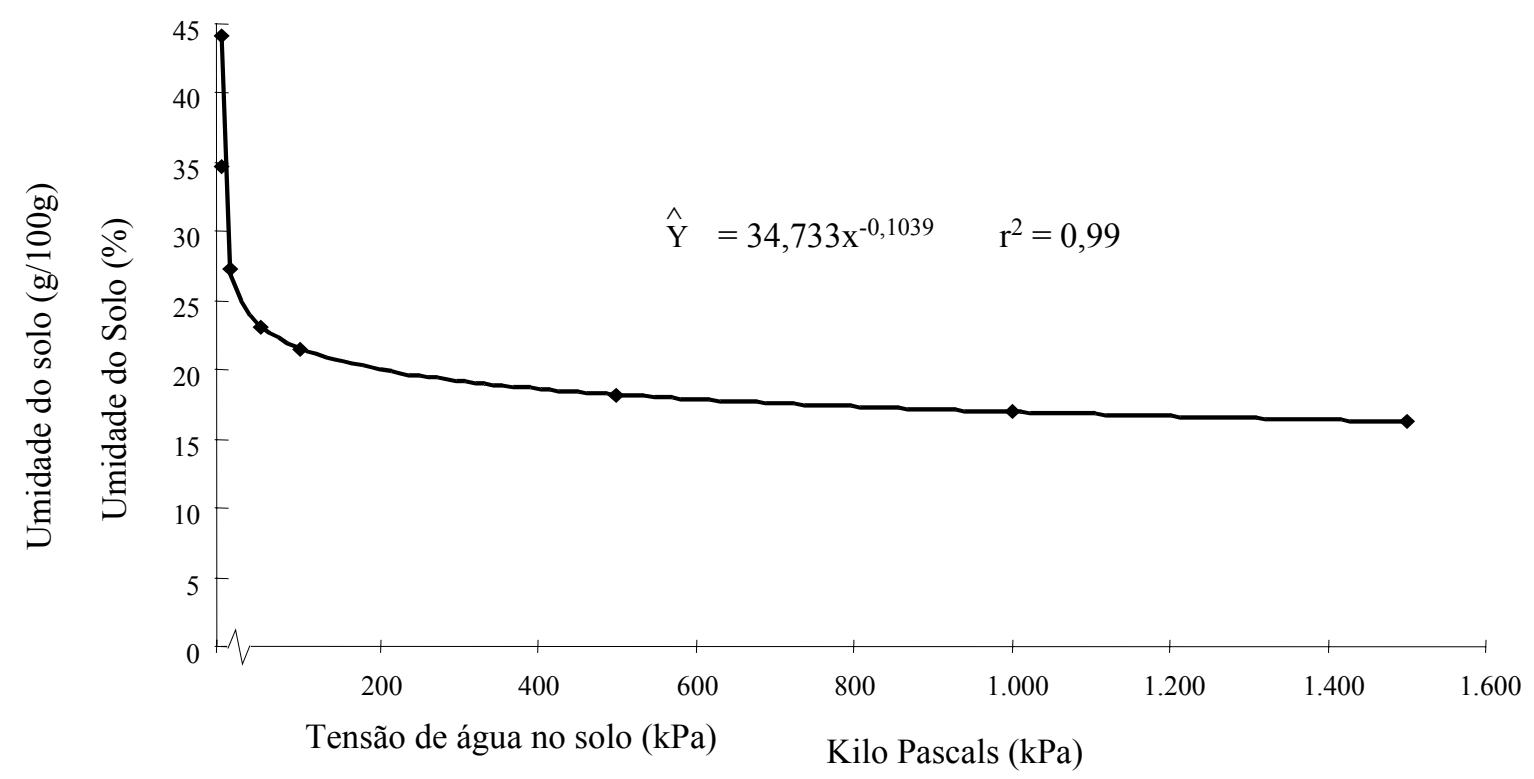

Figura 1: Curva de retenção da água para o solo utilizado

Figure 1: Water retention curve for the soil utilized

Após o preparo do solo, as amostras foram colocadas em vasos de plástico com capacidade para $3,25 \mathrm{~kg}$, os quais foram recobertos internamente com polietileno de $0,05 \mathrm{~mm}$ de espessura, para se evitar a perda de água por percolação. Após determinar os três teores de água no solo nos diferentes tratamentos, conforme a tabela 1, fez-se o transplante das mudas de Eucalyptus citriodora e E. grandis e das mudas de $B$. brizantha, que foram previamente cultivadas em tubetes e caixas de plástico, respectivamente. No momento do transplante, as mudas de eucalipto encontravam-se com aproximadamente $20 \mathrm{~cm}$ de altura, e as de $B$. brizantha, com quatro folhas. O teor de água no solo foi mantido constante, durante toda a condução do experimento (70 dias após o transplante das mudas), através da reposição da água evapotranspirada. Para esse controle utilizou-se um minitanque "classe A", microtensiômetro e pesagens diárias. A água evapotranspirada foi reposta em três períodos diferentes do dia às $8 \mathrm{~h}, 13 \mathrm{~h}$ e $16 \mathrm{~h} 30$. Durante estes períodos foram determinados: evaporação potencial média $(378,4 \mathrm{~mL})$, a temperatura máxima média $\left(34,7^{\circ} \mathrm{C}\right)$, a temperatura mínima média $\left(21,0^{\circ} \mathrm{C}\right)$ e a umidade relativa do ar às $7 \mathrm{~h} 30(83,6 \%)$ e às $14 \mathrm{~h}(56,2 \%)$.

Tabela 1: Umidade residual do solo, tensão de água no solo, porcentagem do teor de água correspondente; e massa de água adicionada por vaso

Table 1: Soil residual moisture, maintained water tension, water moisture percent, and water mass added per pot

\begin{tabular}{|c|c|c|c|}
\hline $\begin{array}{c}\text { Umidade Residual do } \\
\text { solo, antes do } \\
\text { transplante } \\
(\mathrm{g} / \mathrm{g}) \\
\end{array}$ & $\begin{array}{l}\text { Tensão de água no solo } \\
\text { mantido após o } \\
\text { transplante das mudas } \\
(\mathrm{kPa}) \\
\end{array}$ & $\begin{array}{l}\text { Teor de água no solo } \\
\text { mantido após o } \\
\text { transplante das mudas } \\
(\mathrm{g} / \mathrm{g})\end{array}$ & $\begin{array}{l}\text { Quantidade de água } \\
\text { adicionada/vaso e } \\
\text { mantida após o } \\
\text { transplante das mudas } \\
(\mathrm{g})\end{array}$ \\
\hline 0,105 & 202,8 & 0,20 & 250 \\
\hline 0,105 & 49,5 & 0,23 & 325 \\
\hline 0,105 & 16,2 & 0,26 & 400 \\
\hline
\end{tabular}


O experimento foi constituído de 33 tratamentos, compreendendo somente as espécies de eucalipto (E. citriodora e $E$. grandis), três teores de água: condições próximo ao estresse $(20 \%)$; intermediária (23\%); e condições próximo à capacidade de campo (26\%); e quatro quantidades diferentes de $B$. brizantha $(0,1,2$ e 3 plantas/vaso), mais três teores de água em condições próximo ao estresse (20\%); intermediária (23\%); e condições próximo à capacidade de campo $(26 \%)$ e três níveis de competição com $B$. brizantha (1, 2 e 3 plantas/vaso). O espaçamento entre os vasos foi de $35 \mathrm{~cm}$. O delineamento adotado foi o de blocos ao acaso, com quatro repetições, em esquema fatorial $2 \times 3 \times 4+3 \times 3$. Desta forma, em 96 vasos foram plantados uma muda de eucalipto/vaso e em 36 vasos foram plantados, somente $B$. brizantha, totalizando 132 unidades experimentais.

Durante a condução do experimento, fez-se a complementação da fertilização aplicando-se, alternadamente, a cada 7 dias, solução nutritiva de CLARK (1975) e a formulação 20-05-20 (N, P, K). Dessa forma, o total dos nutrientes aplicados por vaso foi de: $278 \mathrm{mg}$ de N; $62 \mathrm{mg}$ de P; $265 \mathrm{mg}$ de K; 31 $\mathrm{mg}$ de $\mathrm{Ca}$ e $5 \mathrm{mg}$ de $\mathrm{Mg}$.

As características avaliadas foram: a) Índice de consumo de água, que é a relação entre a água evapotranspirada e a evaporação potencial, determinadas aos $7,14,21,28,35$, $42,44,56,63$ e 70 dias após o transplante das mudas; b) - Eficiência do uso da água ou coeficiente transpiratório, que é a relação entre a água consumida e a biomassa seca final (aos 70 dias após o transplante das mudas) menos a inicial (média da biomassa das mudas do eucalipto selecionadas, provenientes dos tubetes, no primeiro dia da instalação do experimento). A quantidade de água consumida foi obtida pela diferença entre a água evapotranspirada e a evaporação potencial, sendo determinada por parcela, durante a condução do experimento, por 10 semanas.

Índice de consumo de água (ICA) foi estimado por meio de uma equação de regressão para cada um dos tratamentos, utilizando-se como variável independente, dias após o transplante das mudas $(7,14,28,35$, $42,49,56,63$ e 70). Na análise de regressão, consideraram-se os 10 valores de ICA pareados com o mesmo número de dias após o transplante das mudas, sendo a significância testada com o desvio da regressão, em cada tratamento, utilizando-se o teste $\mathrm{F}$, a $5 \%$ de probabilidade.

Os dados do coeficiente transpiratório, ou eficiência do uso da água, não foram submetidos à análise de variância; foram apenas comparados à literatura.

\section{RESULTADOS E DISCUSSÃO}

\section{Eficiência do uso da água ou coeficiente transpiratório}

E. citriodora, E. grandis e B. brizantha consumiram 17.934, 20.179 e $15.437 \mathrm{~g}$ de água, respectivamente, quando submetidas às condições sob estresse hídrico (20\%). Na medida em que se aumentou o teor de água no solo, o consumo de água pelas plantas se elevou. Assim, em condições próximas à capacidade de campo (26\%), os valores foram 48.015 g para $E$. citriodora, $59.849 \mathrm{~g}$ para $E$. grandis e 33.947 para $B$. brizantha, de forma que as plantas absorveram mais água do solo, o que implicou em maior crescimento e maior produção de biomassa seca, nesse teor de água (tabela 2).

E. grandis destacou-se por apresentar maior consumo de água, em todos os tratamentos, seguido por E. citriodora, verificando-se menor consumo para $B$. brizantha. Observa-se ainda nesta tabela que a eficiência do uso da água ou coeficiente transpiratório para as plantas de E. citriodora, $E$. grandis e $B$. brizantha, praticamente, não variou, quando submetida aos três diferentes teores de água. Esses resultados estão compatíveis com SILVA (1989); Taylor \& Willatt (1983), que reforçam a tese de que a eficiência de uso de água não muda, à medida que a disponibilidade de água varia no solo, indicando ser uma característica das espécies. 
Tabela 2: Valores médios obtidos para a água consumida, biomassa seca, eficiência no uso de água em plantas de eucaliptos cultivadas a 20, 23 e 26\% de água no substrato, em ausência de $B$. brizantha e os valores médios obtidos para $B$. brizantha

Table 2: $\quad$ Mean values obtained for consumed water, dry biomass and water use efficiency of eucalyptus plants at 20, 23 and $26 \%$ of water in B. brizantha absence and, too, mean values obtained for B. brizantha

\begin{tabular}{|c|c|c|c|c|c|c|c|c|c|}
\hline \multirow{4}{*}{$\begin{array}{l}\text { Umidade } \\
\text { do solo } \\
(\%)\end{array}$} & \multicolumn{3}{|c|}{$\begin{array}{l}\text { Água consumida }^{1 /} \\
(\mathrm{g})\end{array}$} & \multicolumn{3}{|c|}{$\begin{array}{l}\text { Biomassa seca }(\mathrm{g}) \\
\text { (final - inicial) }\end{array}$} & \multicolumn{3}{|c|}{$\begin{array}{c}\text { Água consumida }(\mathrm{g}) /(\mathrm{g}) \\
\text { biomassa seca (final - inicial) }\end{array}$} \\
\hline & & $E$ & $B$. & $E$. & & $B$. & $E$. & & $B$. \\
\hline & $E$. & grandi & brizanth & citriodor & E. & brizanth & citriodor & $E$. & brizanth \\
\hline & citriodora & $s$ & $a$ & $a$ & grandis & $a$ & $a$ & grandis & $a$ \\
\hline 20 & 17.934 & 20.179 & 15.437 & 65,89 & 70,89 & 59,41 & 272,2 & 284,7 & 259,8 \\
\hline 23 & 28.694 & 40.229 & 21.793 & 105,91 & 143,84 & 81,81 & 270,9 & 279,7 & 266,4 \\
\hline 26 & 48.015 & 59.849 & 33.947 & 158,90 & 210,86 & 126,93 & 302,2 & 283,8 & 267,4 \\
\hline
\end{tabular}

${ }^{1 /}$ Água consumida $=\underset{i=1}{\sum}$ água evapotranspirada $-\underset{i=1}{\sum} \quad \begin{gathered}\text { água evaporada } \\ \mathrm{i}=1\end{gathered}$

Comparando a eficiência do uso da água entre espécies verifica-se sob estresse hídrico $(20 \%)$, B. brizantha apresentou maior eficiência, isto é, proporcionou menor coeficiente transpiratório $(259,8 \mathrm{~g} / \mathrm{g})$ - grama de água por grama de biomassa seca produzida, seguindo-se E. citriodora com 272,2 g/g e E. grandis com 284,7 g/g. Quando se trabalhou em condições ótimas de água no solo (26\%), verificou-se esta mesma comparação, com exceção de E. citriodora com 302,2 g/g, que passou ser um pouco menos eficiente em relação às condições sob estresse hídrico (tabela 2). Este fato pode explicar o melhor desenvolvimento de $E$. citriodora em condições de pouca disponibilidade de água no solo. De qualquer forma, dentro dos três níveis de água estudados, as duas espécies de eucalipto e $B$. brizantha estão dentro da faixa de plantas eficientes quanto ao uso de água, apesar de o eucalipto ser planta $C_{3}$ Ludlow (1980) e $B$. brizantha $\mathrm{C}_{4}$ Duke (1987).

Estes resultados são explicados porque a eficiência no uso da água ou o coeficiente transpiratório varia de acordo com as espécies de plantas. As plantas $\mathrm{C}_{4}$ são mais eficientes que as $\mathrm{C}_{3}$ na economia da água. As estimativas da eficiência do uso da água nas plantas $\mathrm{C}_{4}$ variam de 200 a 333 g de água por grama de biomassa seca produzida e nas plantas $C_{3}$ varia de 333 a 500 g de água por grama de biomassa seca produzida dependendo do ambiente evaporativo Briggs \& Shantz (1914); Neales et al. (1968); Taylor \& Willatt (1983); Kramer \& Boyer (1995). Segundo Beadle et al. (1985), o coeficiente transpiratório varia de 450 a 950 para as plantas $\mathrm{C}_{3}$ e 250 a 350 para as plantas $\mathrm{C}_{4}$. Para Silva et al. (2000a), as plantas $\mathrm{C}_{4}$ são consideradas eficientes quanto ao uso da água e a $\mathrm{C}_{3}$ ineficientes.

\section{Índice de consumo de água}

$\mathrm{O}$ índice de consumo de água no eucalipto variou em função da disponibilidade de água no solo (figura 2). Aos 70 dias após o transplante de mudas, em ausência de $B$. brizantha, E. citriodora e E. grandis alcançaram os maiores ICA, em condições ótimas de água (26\%) seguidos pelo teor intermediário (23\%), enquanto que os menores ICA foram registrados sob condições de estresse hídrico (20\%), Também, nesse teor de água, houve menor produção de biomassa seca, para as duas espécies de eucalipto (tabela 2).

A menor produção de biomassa seca para as duas espécies de eucalipto, nas condições de estresse hídrico, pode estar relaciona com o período de convivência das plantas durante a condução do experimento, que foi na estação do verão, sob evaporação potencial de $378,4 \mathrm{~mL}$ e temperatura máxima média de $34,7^{\circ} \mathrm{C}$. Neste contexto, estes dois fenômenos juntamente com a incidência da radiação solar deveria ter possibilitado 
alterações no metabolismo do eucalipto, em virtude da menor disponibilidade de água (20\%), proporcionando-se assim menor crescimento das plantas; apesar de a água ser reposta três vezes ao dia para manter a umidade desejada.

Para os tratamentos em que $E$. citriodora ou $E$. grandis estavam competindo com uma, duas e três plantas de $B$. brizantha (figura 2), o comportamento do ICA do eucalipto foi semelhante ao obtido em ausência da braquiária. Contudo, maiores valores de ICA foram obtidos a $26 \%$ de água, e os menores valores a $20 \%$. Para os tratamentos que continham somente braquiária com uma, duas e três plantas por vaso (figura 3), o aumento das plantas por vaso, praticamente, não afetou o ICA. Segundo Gholz et al. (1990), a disponibilidade de água afeta o crescimento das plantas, por controlar a abertura dos estômatos e, consequentemente, a atividade fotossintética e produção de biomassa seca. Um decréscimo de água no solo diminui o potencial de água na folha e na condutância estomática, promovendo $\mathrm{o}$ fechamento dos estômatos. Esse fechamento bloqueia o influxo de $\mathrm{CO}_{2}$ para as folhas, afetando o acúmulo de fotoassimilados, o que implica redução da produtividade.

Plantas que reduzem rapidamente a condutância estomática são consideradas preventivas, enquanto espécies que mantêm os estômatos abertos, quando submetidos a déficits hídricos severos são tidas como tolerantes à seca Levitt (1980). Diante disso, poder-se-ia considerar E. grandis como uma espécie que previne a seca, visto que seus estômatos se fecham antes que algum murchamento visível ocorra, enquanto $E$. camaldulensis parece tolerar a desidratação hídrica Façanha (1983). De acordo com Goes (1962), as espécies de eucalipto, quanto ao grau de resistência à seca são divididas em: muito resistentes, resistência média e pouco resistentes. Em primeiro lugar estão $E$. camaldulensis e E. tereticornis, em segundo $E$ grandis e, em terceiro, E. citriodora.

$\mathrm{O}$ estresse hídrico promoveu as maiores reduções na biomassa seca e na área foliar das plantas de E. citriodora e E. grandis
Silva et al. (1999). Esse comportamento tem sido relatado, para diversas espécies de Eucalyptus spp., por diferentes pesquisadores Myers \& Landsberg (1989); Stoneman et al. (1994). À medida que se aumentou a disponibilidade hídrica no solo, as plantas de $E$. grandis e $E$. citriodora, em ausência de $B$. brizantha, apresentaram valores semelhantes. Maiores ICA, em ausência de $B$. brizantha, em relação à presença, estão relacionados a maior área foliar e produção de biomassa, conforme pesquisa apresentada por Silva et al. (1999).

De acordo com Balloni \& Simões (1980), na fase de estabelecimento, água, nutrientes e luz podem limitar o crescimento das plantas. A intensidade de competição por esses fatores ambientais depende da densidade populacional do eucalipto e da comunidade infestante de plantas daninhas Silva (1993). Segundo Viets (1962), o maior desenvolvimento pode levar a uma exaustão mais rápida da água do solo, por aumentar a demanda hídrica na planta. Conforme explica Deuber (1992), a presença das plantas daninhas nas áreas cultivadas resulta na redução da produtividade, tanto pela competição direta pelos fatores de produção, quanto pela interferência sobre as plantas cultivadas, como ocorre no caso da alelopatia. Segundo Silva et al. (2000b), E. grandis obteve maior altura e diâmetro; de acordo com Silva et al. (1999), maior biomassa seca, quando comparado com E. citriodora, tanto em presença de uma, quanto de duas e de três plantas de B. brizantha por vaso, apesar de o ICA de E. citriodora ser maior que o de $E$. grandis, em presença de uma e duas gramíneas por vaso. Menor altura e diâmetro das espécies de eucalipto foram apresentados, quando estavam em convivência com $B$. brizantha, foi relacionado com menor taxa da fotossíntese líquida (Silva et al., 2001). Ferri (1985) constatou que a taxa de fotossíntese líquida em condições de saturação de luz, para as plantas $\mathrm{C}_{3}$, varia de $15-35 \mathrm{mg} \mathrm{CO} \mathrm{dm}^{-}{ }^{-1}$; enquanto que nas plantas $\mathrm{C}_{4}$ esse valor varia de 40-80 $\mathrm{mg} \mathrm{CO}_{2} \mathrm{dm}^{-2} \mathrm{~h}^{-1}$. Pode-se ressaltar que $\mathrm{o}$ eucalipto é uma planta $C_{3}$ Ludlow (1980) e $B$. brizantha uma planta $\mathrm{C}_{4}$ Duke (1987). 


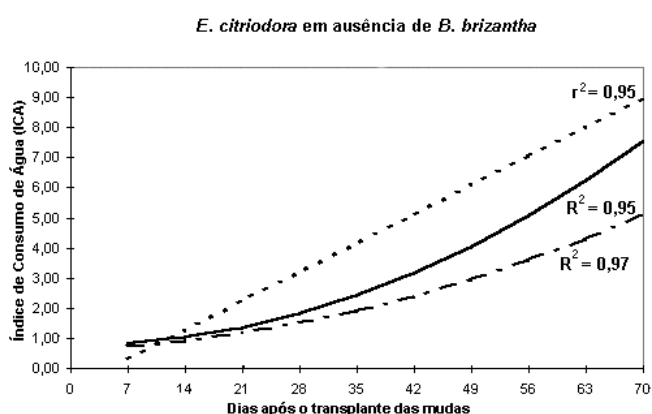

E. citriodora em presença de 1 planta por vaso de $B$. brizantha

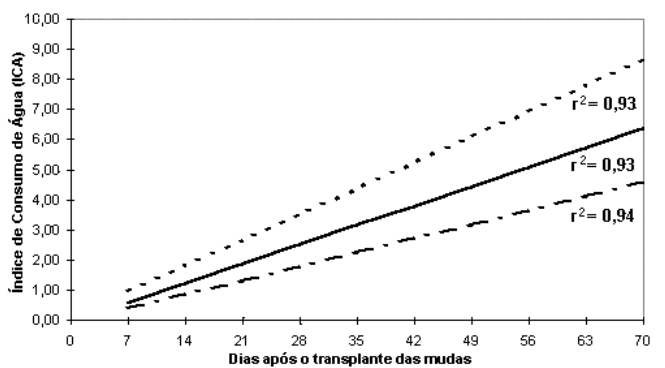

E. citriodora em presença de 2 plantas por vaso de $B$. brizantha

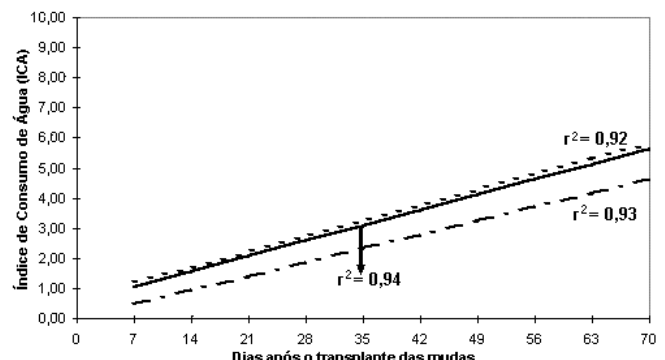

E. citriodora em presença de 3 plantas por vaso de $B$. brizantha

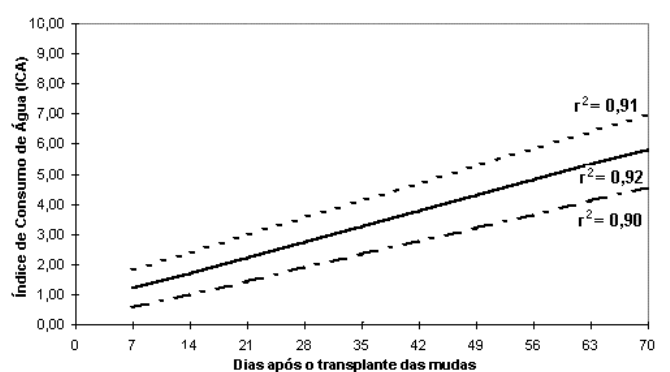

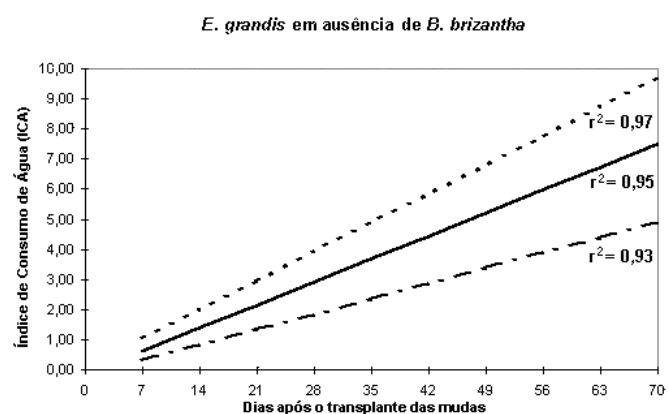

E. grandis em presença de 2 plantas por vaso de $B$. brizantha

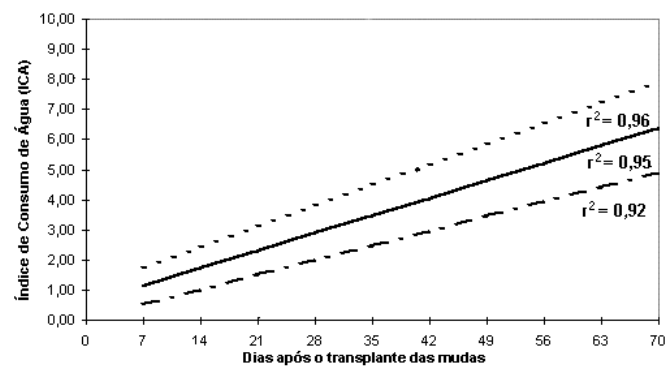

E. grandis em presença de 2 plantas por vaso de $B$. brizantha

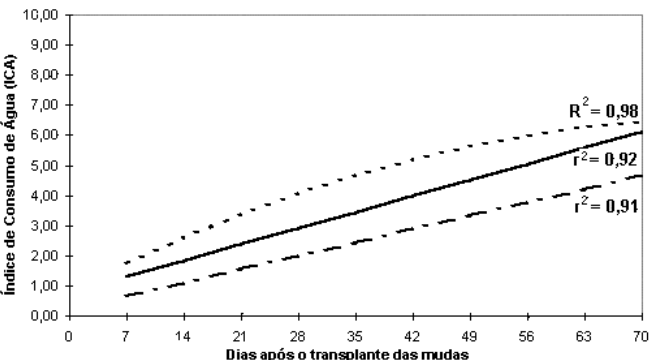

E. grandis em presença de 3 plantas por vaso de $B$. brizantha

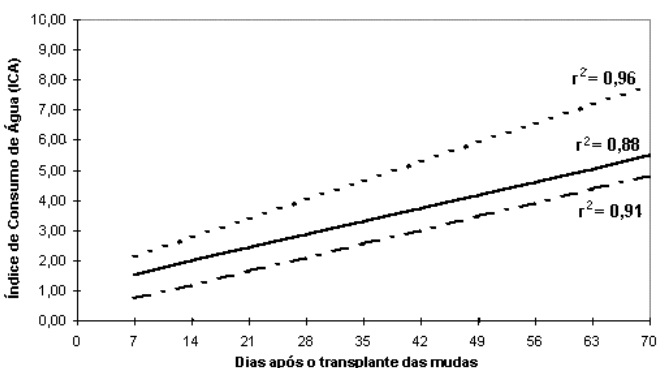

$\cdots \cdot 26 \%$

Figura 2: Índice de consumo de água (ICA) de E. citriodora e E. grandis, em ausência e em presença de 1, 2 e 3 plantas de B. brizantha por vaso, a 20,23 e $26 \%$ de água, no solo, durante dez semanas, após o transplante das mudas

Figure 2: E. citriodora e E. grandis water consumption index (wci) in absence and presence of 1,2 and 3 plants de B. brizantha/pot, at 20, 23 and $26 \%$ of water in the soil, during tem weeks after seedlings' transplantation 
Figura 2 - cont.,

E. citriodora - Ausência de B. brizantha

- - $\hat{\mathrm{Y}}=0,6254+0,009492 * * \mathrm{x}+0,0007765 * \mathrm{x}^{2}$

$\hat{\mathrm{Y}}=0,7993-0,004549 * * \mathrm{x}+0,001440 * \mathrm{x}^{2}$

-.. $\hat{\mathrm{Y}}=-0,6523+0,1375^{* *} \mathrm{x}$

\section{E. citriodora em Presença 1 B. brizantha}

- - $\quad \hat{\mathrm{Y}}=-0,07450+0,06631 * * \mathrm{x}$

$-\hat{\mathrm{Y}}=-0,06850+0,09167 * * \mathrm{x}$

$-. \cdot-\hat{\mathrm{Y}}=0,09500+0,1222 * * \mathrm{x}$

E. citriodora em Presença de 2 B. brizantha

- - $\quad \hat{\mathrm{Y}}=0,01567+0,06585 * *^{\mathrm{X}}$

$\underline{\hat{Y}}=0,5513+0,07256^{* *} \mathrm{X}$

$-. \cdot-\hat{\mathrm{Y}}=1,1018+0,09173 * *_{\mathrm{x}}$

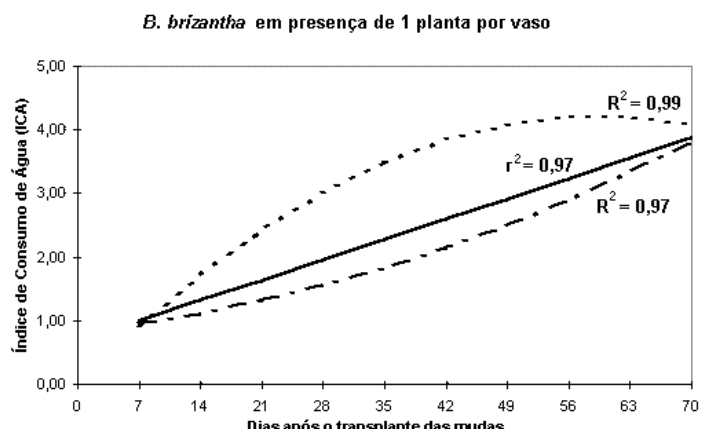

E. grandis - Ausência de B. brizantha

- - $\quad \hat{\mathrm{Y}}=-0,2091+0,07306^{* *} \mathrm{x}$

$-\hat{\mathrm{Y}}=-0,1560+0,1093 * * \mathrm{X}$

$-. .-\hat{\mathrm{Y}}=0,08083+0,1372 * * \mathrm{x}$

E. grandis em Presença 1 B. brizantha

- . $\quad \hat{\mathrm{Y}}=0,0308+0,06967 * * \mathrm{x}$

$-\hat{\mathrm{Y}}=0,5627+0,08283 * *_{\mathrm{x}}$

-..- $\hat{\mathrm{Y}}=1,0507+0,09810^{* * \mathrm{x}}$

E. grandis em Presença de $2 B$. brizantha

- . $\quad \hat{\mathrm{Y}}=0,2133+0,06349 * *_{\mathrm{x}}$

$\underline{\mathrm{Y}}=0,7518+0,07663^{* * \mathrm{x}}$

$-. .-\hat{\mathrm{Y}}=0,7997+0,1404 * * \mathrm{x}-0,0008529 * * \mathrm{x}^{2}$

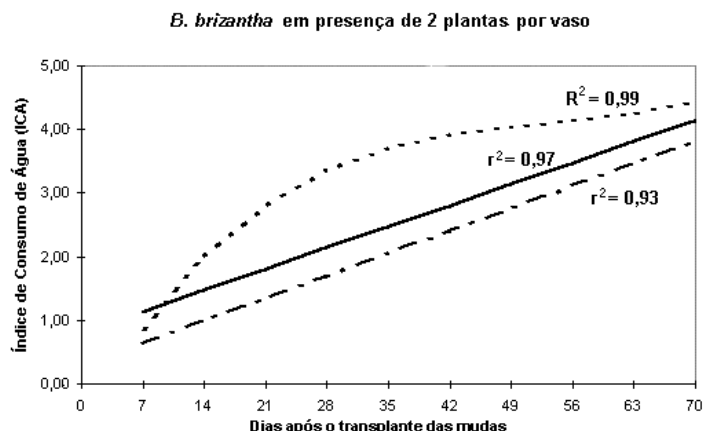

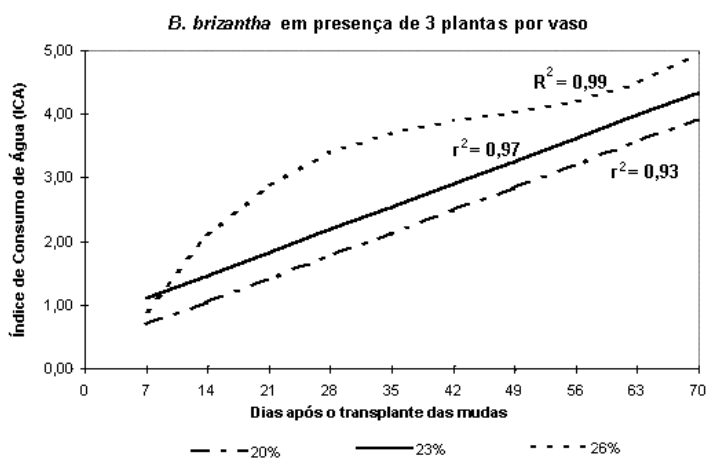

Figura 3: Índice de consumo de água (ICA) de B. brizantha, com 1, 2 e 3 plantas por vaso, a 20, 23 e $26 \%$ de água, no solo, durante dez semanas, após o transplante das mudas

Figure 3: B. brizantha water consumption index (WCI) in association with 1, 2 and 3 plants per pots, at 20, 23 and $26 \%$ of water in the soil, during tem weeks after seedlings' transplantation 
Figura 3 - cont.,

$$
\begin{array}{cc}
\text { Presença de 1 B. brizantha } & \text { Presença de 2 B. brizantha } \\
---\hat{\mathrm{Y}}=0,8290+0,01465^{* *} \mathrm{x}+0,0003964 * * \mathrm{x}^{2} & ---\hat{\mathrm{Y}}=0,2822+0,05046^{* * \mathrm{x}} \\
-\hat{\mathrm{Y}}=0,6743+0,04558^{* *} \mathrm{x} & -\hat{\mathrm{Y}}=0,8030+0,04760^{* * \mathrm{x}} \\
-\cdot-\hat{\mathrm{Y}}=-0,06963+0,1440 * * \mathrm{x}-0,001211^{* *} \mathrm{x}^{2} & -. \cdot \hat{\mathrm{Y}}=-0,6301+0,2418^{* *} \mathrm{x}-0,004325^{* *} \mathrm{x}^{2}+ \\
& 0,00002721^{* *} \mathrm{x}^{3}
\end{array}
$$

Presença de 3 B. brizantha:

$$
\begin{aligned}
& --\hat{\mathrm{Y}}=0,3310+0,05131^{* *} \mathrm{x} \\
& -\hat{\mathrm{Y}}=0,7367+0,05141^{* *} \mathrm{x} \\
& -. \cdot \hat{\mathrm{Y}}=-0,6832+0,2641^{* *} \mathrm{x}-0,005302^{* *} \mathrm{x}^{2}+0,00003828^{* *} \mathrm{x}^{3}
\end{aligned}
$$

\section{CONCLUSÕES}

O maior crescimento de E. citriodora, $E$ grandis e $B$. brizantha foi observado em solos com condições próximo à capacidade de campo $(26 \%)$

A convivência de $B$. brizantha com $E$. citriodora ou $E$. grandis reduziu o acúmulo de biomassa seca do eucalipto, independente do teor de água no solo.

A eficiência do uso da água pelo eucalipto (coeficiente transpiratório) foi alta (282 g de água/g de matéria seca produzida), semelhante ao observado para $B$. brizantha, ambos dentro da faixa de plantas eficientes ao uso de água, independente do teor de água no solo.

\section{REFERÊNCIAS}

BALLONI, E.A., SIMÕES, J.W. O espaçamento de plantio e suas implicações silviculturais. IPEF. v.1, n.3, p.1-16, 1980. (Série Técnica).

BARROS, N.F., NOVAIS, R.F. Relações solo-eucalipto. Viçosa, MG, Folha de Viçosa, 1990. 330p.

BEADLE, C. L., LONG, S.P., IMBAMBA, S.K. Photosynthesis in relation to plant production in terrestrial environments.
Oxford, United Nations Environment Programme, 1985. 156p.

BRIGGS, L.J., SHANTZ, H.L. Relative water requirement of plants. J. Agric. Res., v.3, p.163, 1914.

CLARK, R.B. Characterization of phosphatase of intact maize roots. J. Agric. Food. Chem., v.23, p.458-460, 1975.

DEFELIPO, B. V., RIBEIRO, A.C. Análise química do solo (metodologia). Viçosa, MG: UFV, Imp. Univ., 1981. 17p. (Boletim de extensão, 29).

DENNIS, D.T., TURPIN, D.H. Plant physiology, biochemistry and molecular biology. King Ston, Longman Scientific \& Technical, 1990. 529p.

DEUBER, R. Ciências das plantas daninhas: fundamentos. Jaboticabal, FUNEP, 1992. $431 \mathrm{p}$.

DUKE, S.O. Weed physiology, reproduction and ecophysiology. Boca Raton, CRC, 1987. v.1, 157p.

EMPRESA BRASILEIRA DE PESQUISA AGROPECUÁRIA - EMBRAPA. Manual de métodos de análise de solo. 2.ed. Rio de Janeiro, 1997. 212p.

FAÇANHA, J.G.V. Aspectos fisiológicos do crescimento de Eucalyptus spp. submetidos 
à deficiência hídrica. Viçosa, UFV, 1983. 47p. Tese (Mestrado em Fisiologia Vegetal) Universidade Federal de Viçosa, 1983.

FERRI, M.G. Fisiologia vegetal. São Paulo, EPU, 1985. 362p.

GHOLZ, H.L., EWEL, K.C., TESKEY, R.O. Water and forest productivity. For. Ecol. Manag., v.30, n.1, p.1-18, 1990.

GOEDERT, W.J., LOBATO, E., WAGNER, E. Potencial agrícola da região dos cerrados brasileiros. Pesq. Agropec. Bras., v.15, n.1, p.1-17, 1980.

GOES, E. Os eucaliptos em Portugal: ecologia, cultura e produções. Lisboa, Ministério da Economia, 1962. v.2, p.47-58.

GOMES, R.T. Efeito do espaçamento no crescimento e nas relações hídricas de Eucalyptus spp. na região de cerrado de Minas Gerais. Viçosa, UFV, 1994. 85p. Tese (Mestrado em Ciência Florestal) Universidade Federal de Viçosa, 1994.

JONES, D.A., WILKINS, D.A. Variation and adaptation in plant species. London, Heinemann, 1971. 184p.

KRAMER, P. J., BOYER, J.S. Water relations of plants and soil. New York, Academic, 1995. 495p.

LEVITT, J. Responses of plants to environmental stress: water, radiation, salt, and other stresses. New York, Academic, 1980. v.2, p.25-280.

LUDLOW, M.M. Adaptive significance of stomatal responses to water stress. In: TURNER, N.C., KRAMER, P.J. (Eds.). Adaptation of plants to water and high temperature stress. New York, John-Wiley, 1980. p.123-138.

MYERS, B.J., LANDSBERG, J.J. Water stress and seedlings growth of two eucalypti species from contrasting habitats. Tree Physiol., 5(2):207-218, 1989.

NEALES, T.F., PATTERSON, A.A., HARTNEY, V.J. Physiological adaptation to drought in the carbon assimilation and water loss of xeroplayter. Nature, v.219, p.469-472, 1968.
NOVAIS, R.F, BARROS, N.F., NEVES, J.C.L. Nutrição mineral de mudas de eucalipto. In: BARROS, N.F., NOVAIS, R.F. (Eds.). Relação solo-eucalipto. Viçosa, MG, Folha de Viçosa, 1990. p.25-98.

OLBRICH, B.W., ROUX, D.LE, POULTER, A.G. Variation in water use efficiency and G13C levels in Eucalyptus grandis clones. J. Hydrology, v.150, p.615-633, 1993.

PRYOR, L.D. The biology of eucalyptus. London, Edward Arnold, 1976. 82p.

RICHARDS, L.A. Methods of measuring soil moisture tension. Soil Sci., v.15, p.95-112, 1949.

SILVA, A.A. Bioatividade do alachlor e do metribuzin sob diferentes manejos de água no solo, e efeito do metribuzin, sob estas condições, em soja (Glycine max (L.) Merril). Piracicaba, ESALQ, 1989. 138p. Tese (Doutorado em Fitotecnia) - Escola Superior Luiz de Queiroz, 1989.

SILVA, A. A., SILVA, J.F., FERREIRA, F.A., FERREIRA, L.R., FERREIRA, J.F. Associação Brasileira de Educação Agrícola Superior. ABEAS. Curso de proteção de plantas. Módulo,3 - Brasília, DF: ABEAS; Viçosa, MG: UFV, 2000 (a). 260p.

SILVA, W, SILVA, A.A., SEDIYAMA, T. ANTÔNIO, A.C. Biomassa e área foliar em mudas de Eucalyptus citriodora e E. grandis, em resposta a diferentes teores de água no solo e convivência de Brachiaria brizantha. Pesquisa Agrop. Gaúcha, v.5,n.1, p. 29-36, 1999.

SILVA, W, SILVA, A.A., SEDIYAMA, T., FREITAS, L.H.L. Altura e diâmetro de Eucalyptus citriodora e E. grandis, submetidos a diferentes teores de água em convivência com Brachiaria brizantha. Floresta, v.27,n.1/2, p. 3-16, 2000 (b).

SILVA, W, SEDIYAMA, T., SILVA, A.A., SOUZA, A.P. Taxa fotossintética líquida de Eucalyptus citriodora Hook e E. grandis W. Hill em resposta à diferentes níveis de água no solo e associação com Brachiaria brizantha Stapf. Acta Scientiarum, v.23,n.5, p. 12051209, 2001. 
SILVA, W. Tolerância de Eucalyptus spp. a herbicidas e a eficiência desses produtos no controle de plantas daninhas. Viçosa, UFV, 1993. 86p. Tese (Mestrado em Fitotecnia) Universidade Federal de Viçosa, 1993.

STONEMAN, G.L., TURNER, N.C., DELL, B. Leaf growth, photosynthesis and tissue water relations of greenhouse-growth Eucalyptus marginata seedlings in response to water deficits. Tree Physiol., v.14, p.633-646, 1994.
TAYLOR, H. M., WILLATT, S.T. Shrinkage of soybean roots. Agron. J., v.75, p.818-820, 1983.

VALE, F. R. Efeito do alumínio sobre a cinética de absorção de nitrato, amônio e fosfato em milho (Zea mays, L.) e em clone de eucalipto (Eucalyptus alba). Viçosa, MG: UFV, 1982. 71p. Tese (Mestrado em Fitotecnia) - Universidade Federal de Viçosa, 1982.

VIETS, F.G. Fertilizers and efficient use of water. Adv. Agron., v.14, p.223-264, 1962. 